\title{
Ebola viral load at diagnosis associates with patient outcome and outbreak evolution
}

\author{
Marc-Antoine de La Vega, ${ }^{1,2}$ Grazia Caleo, ${ }^{3}$ Jonathan Audet, ${ }^{1,4}$ Xiangguo Qiu, ${ }^{1,4}$ Robert A. Kozak, ${ }^{1}$ James I. Brooks, ${ }^{5}$ Steven Kern, ${ }^{6}$ \\ Anja Wolz, ${ }^{7}$ Armand Sprecher, ${ }^{8}$ Jane Greig, ${ }^{3}$ Kamalini Lokuge, ${ }^{9}$ David K. Kargbo, ${ }^{10}$ Brima Kargbo, ${ }^{10}$ Antonino Di Caro, ${ }^{11}$ \\ Allen Grolla, ${ }^{1}$ Darwyn Kobasa, ${ }^{1}$ James E. Strong, ${ }^{1}$ Giuseppe Ippolito, ${ }^{11}$ Michel Van Herp, ${ }^{7}$ and Gary P. Kobinger ${ }^{1,2,4,12}$

\begin{abstract}
'Special Pathogens Program, National Microbiology Laboratory, Public Health Agency of Canada (PHAC), Winnipeg, Manitoba, Canada. ²Department of Immunology, University of Manitoba, Winnipeg, Manitoba, Canada. ${ }^{3}$ Manson Unit, Médecins Sans Frontières (MSF), London, United Kingdom. ${ }^{4}$ Department of Medical Microbiology, University of Manitoba, Winnipeg, Manitoba, Canada. ${ }^{5}$ National Laboratory for HIV Genetics, PHAC, Ottawa, Ontario, Canada. ${ }^{6}$ Quantitative Sciences, Bill and Melinda Cates Foundation, Seattle, Washington, USA. ${ }^{7}$ Medical Department Unit and ${ }^{8}$ Operational Centre (Brussels), MSF, Brussels, Belgium. ${ }^{9}$ National Centre for Epidemiology and Population Health, Research School of Population Health, Australian National University, Canberra, Australia. ${ }^{10}$ Ministry of Health $(\mathrm{MoH})$ and Sanitation, Government of Sierra Leone, Freetown, Sierra Leone. "Lazzaro Spallanzani National Institute for Infectious Diseases, Rome, Italy.
\end{abstract} \\ ${ }^{12}$ Department of Pathology and Laboratory Medicine, University of Pennsylvania School of Medicine, Philadelphia, Pennsylvania, USA.
}

BACKGROUND. Ebola virus (EBOV) causes periodic outbreaks of life-threatening EBOV disease in Africa. Historically, these outbreaks have been relatively small and geographically contained; however, the magnitude of the EBOV outbreak that began in 2014 in West Africa has been unprecedented. The aim of this study was to describe the viral kinetics of EBOV during this outbreak and identify factors that contribute to outbreak progression.

METHODS. From July to December 2014, one laboratory in Sierra Leone processed over 2,700 patient samples for EBOV detection by quantitative PCR (qPCR). Viremia was measured following patient admission. Age, sex, and approximate time of symptom onset were also recorded for each patient. The data was analyzed using various mathematical models to find trends of potential interest.

RESULTS. The analysis revealed a significant difference $\left(P=2.7 \times 10^{-77}\right)$ between the initial viremia of survivors $\left(4.02 \log _{10}\right.$ genome equivalents [GEQ] $/ \mathrm{ml}$ ) and nonsurvivors $\left(6.18 \log _{10} \mathrm{GEQ} / \mathrm{ml}\right)$. At the population level, patient viral loads were higher on average in July than in November, even when accounting for outcome and time since onset of symptoms. This decrease in viral loads temporally correlated with an increase in circulating EBOV-specific IgC antibodies among individuals who were suspected of being infected but shown to be negative for the virus by PCR.

CONCLUSIONS. Our results indicate that initial viremia is associated with outcome of the individual and outbreak duration; therefore, care must be taken in planning clinical trials and interventions. Additional research in virus adaptation and the impacts of host factors on EBOV transmission and pathogenesis is needed.

\section{Introduction}

Ebola virus (EBOV) $(1,2)$ is one of the deadliest pathogens in existence. Infection of a suitable host results in EBOV disease (EVD), which is characterized by the onset of a broad array of flu-like symptoms including fever, myalgia, and a general malaise. Disease progression leads to gastrointestinal manifestations, multiple-organ failure, and eventual death in up to $90 \%$ of cases $(3,4)$. On March 22, 2014, WHO was notified by the $\mathrm{MoH}$ of Guinea that an outbreak of EVD

Role of funding source: The PHAC had no role in study design, data collection, or data analysis.

Authorship note: Marc-Antoine de La Vega and Grazia Caleo contributed equally to this work.

Conflict of interest: The authors have declared that no conflict of interest exists Submitted: June 8, 2015; Accepted: September 28, 2015.

Reference information: J Clin Invest. 2015;125(12):4421-4428. doi:10.1172/JCI83162. was rapidly unfolding in the south-eastern region of the country, near the borders of Sierra Leone and Liberia (5). Genomic characterization of the virus identified it as a novel EBOV variant (strain Makona), with notable sequence heterogeneity compared with the 1995 Kikwit reference strain (5-7). Subsequently, Liberia reported its first confirmed case (8), and by the end of May 2014 , Sierra Leone was also adding its name to the list of affected countries (9). Over a year has passed since the beginning of the largest documented Ebola outbreak, and to date (as of August 12,2015 ) there have been 27,948 cases (reported and confirmed) and 11,284 deaths. Disconcertingly, Sierra Leone has accounted for almost $50 \%$ of the case burden.

This study investigated the temporal changes in viral loads among laboratory-confirmed cases that were presented at the Kailahun Ebola Management Centre during a 5-month period of the outbreak and highlighted key factors that may have influenced the progression of the epidemic. 

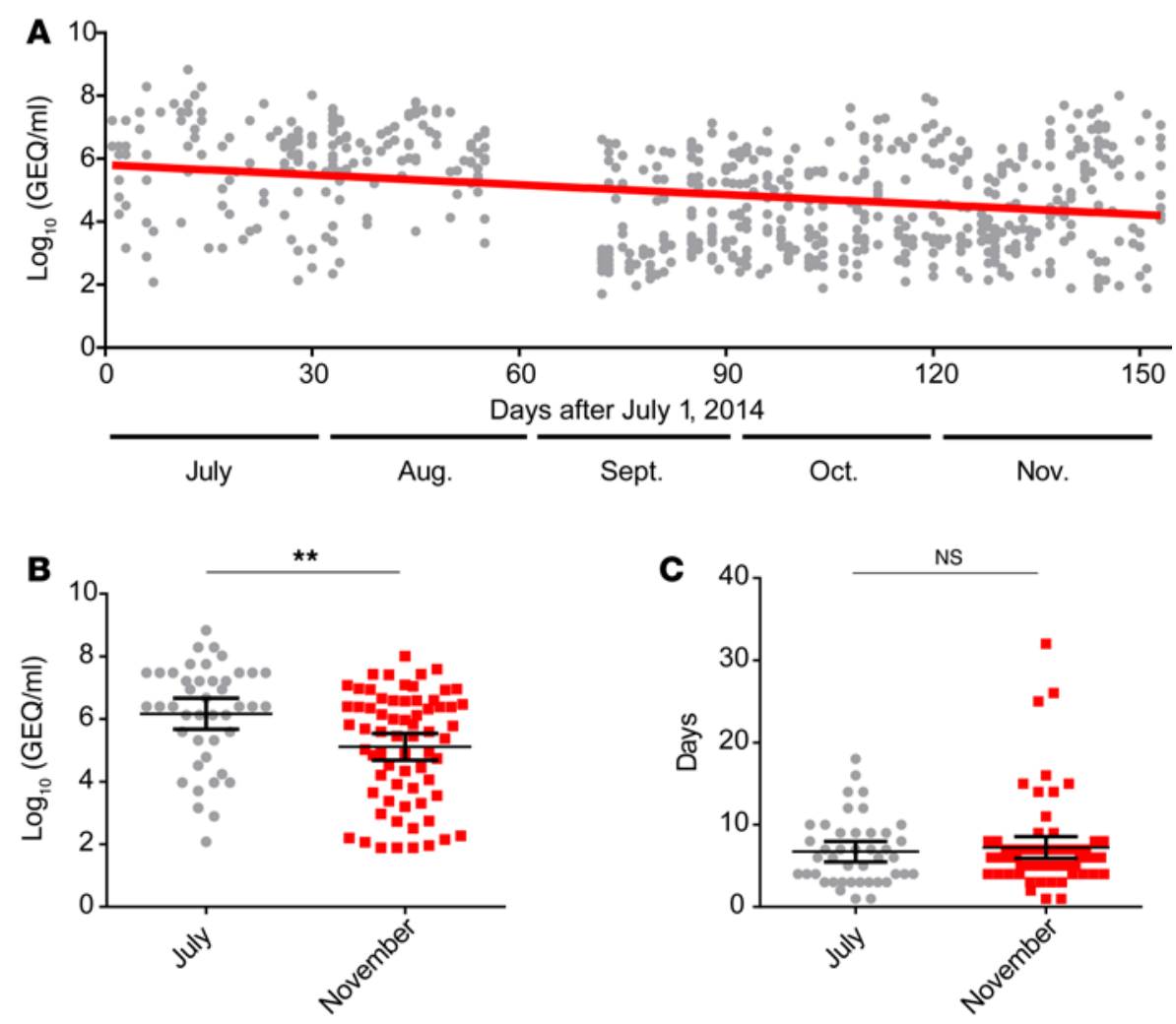

\section{Results}

Characteristics of the study population. Approximately 1,200 people were tested for EBOV infection at the Kailahun Ebola Management Center (EMC), of which 632 had a complete data set and were included in the analysis. The data set consisted of: age, sex, date of symptom onset, dates of all available sampling, viral load, and patient outcome (deceased or survived). Individuals without a complete data set were excluded from the analysis. The median age of the study population was 25 years old (mean: 26.9 years old; range: 4 months -75 years old). The population was composed of 319 males and 313 females. Our dataset included 379 survivors and 253 fatalities for an overall case fatality rate (CFR) of $40 \%$. The median time between symptom onset and diagnostic sampling was 7 days, with an interquartile range of 6 days. The characteristics of the cohort studied are summarized in Table 1.

Bivariable analyses. We initially compared viremia across the different aspects of the population, such as age, sex, and survivorship. Nonsurvivors had a significantly higher viremia on admission (6.18 $\log _{10}$ genome equivalents [GEQ] $/ \mathrm{ml}$ of blood, 95\% CI, 6.04-6.32) compared with survivors (4.02 $\log _{10}$ GEQ/ml of blood, 95\% CI, 3.89-4.15) $\left(P=2.70 \times 10^{-77}\right)$ (Table 1). Age $(P=0.0104)$ and time between symptom onset and sampling $\left(P=5.63 \times 10^{-15}\right)$ were also significantly associated with viremia. There was no difference in initial viremia between the sexes.

Outbreak kinetics. We first sought to determine if the initial viral loads of patients upon admission to the EMC changed over the course of the outbreak, regardless of outcome. The viral loads of 632 patients who tested positive for EBOV immediately following presentation at the EMC were plotted over the course of the epidemic (Figure 1A) and fitted to a linear regression. Analysis of this data indicated that the viral loads of patients following arrival at the EMC were lower at
Figure 1. Characterization of the viral load levels. When applicable, the arithmetic mean is shown, and the error bars represent the $95 \%$ Cl. (A) Initial viral loads of the selected 632 patients throughout the outbreak in Kailahun, Sierra Leone. Linear regression is shown in red. $(n=632)$. (B) Initial viral loads of patients for the first 2 weeks of July $(n=43)$ and the last 2 weeks of November $(n=67)$. (c) Number of days between the reported date of symptom onset and the date of the initial sampling at the EMC on the same data set from $\mathbf{B}$. Two-tailed, unpaired $t$ tests. ${ }^{* *} 0.001<P<0.01$.

the end of the data set in November when compared with July (slope $=-0.01051[95 \%$ CI, -0.01336 to -0.007661$] \log _{10}$ GEQ/ml per day). The mobile laboratory operated by the PHAC was not active between August 25 and September 9, 2014, (epidemiological weeks 35-37), since the personnel was evacuated for safety reasons, explaining the gap in coverage for this time period (Figure 1A). However, the same equipment, reagents, and protocols were used before and after the evacuation. Many of the laboratory staff deployed from September to November had also been deployed in July or August. Staff members reported similar performance for internal controls before and after the gap.

A decrease of the mean initial viremia was observed when samples collected at the beginning of this study (first 2 weeks of July, when the EMC was initially opened) were compared with those taken immediately prior to the closing of the facility (final 2 weeks of November). The mean initial viremia for July was $6.168 \log _{10}$ GEQ/ $\mathrm{ml}$ of blood (95\% CI, 5.673-6.662), but had dropped to $5.110 \log _{10}$ GEQ/ml of blood (95\% CI, 4.681-5.538) by November (Figure 1B). An unpaired, 2-tailed $t$ test revealed this difference to be significant $(\mathrm{t}[108]=3.184, P=0.0019)$. To understand potential factors that may have played a role in the observed decrease in viral loads, we first compared the difference in days between the reported date of disease onset and the date of initial sampling. The analysis revealed that patients in July arrived to the EMC, on average, 6.7 (95\% CI, 5.5-8.0) days after the reported onset of symptoms. This average was $7.2(95 \%$ CI, 5.9-8.6) days for November (Figure 1C). An unpaired, 2-tailed $t$ test indicated this difference to be nonsignificant $(\mathrm{t}[108]=0.5220$, $P=0.6028)$. Linear regression analysis revealed that, from July 1 to July 31 , there was a nonsignificant 0.22 decrease in $\log _{10}$ GEQ/ml of blood [95\% CI, -1.25-0.81]. Similarly, a nonsignificant $0.31 \mathrm{log}$ increase in viral loads (95\% CI, -0.48-1.10) was observed between July 1 and August 31. However, marked decreases were observed when the analysis spanned from July 1 to September $30(-2.37$ log-units, 95\% CI, -2.90 to -1.84$)$, October 31 (-2.08 log-units; $95 \%$ CI, -2.56 to -1.61 ), and November 30 (-1.60 log-units; 95\% CI, -2.04 to -1.17 ) (Supplemental Table 2; supplemental material available online with this article; doi:10.1172/JCI83162DS1).

Viral load as a predictor of outcome. We next investigated whether age and viral loads at admission had an impact on patient outcome that changed during the outbreak. To this end, we categorized the 
Table 1. Descriptive and bivariable analysis of the patients

\begin{tabular}{|c|c|c|c|c|c|}
\hline \multicolumn{3}{|c|}{ Subjects } & \multicolumn{3}{|c|}{ EBOV viremia on admission ( $\left.\log _{10} \mathrm{CEQ} / \mathrm{ml}\right)$} \\
\hline Feature & & $\#$ & Mean $[95 \% \mathrm{Cl}]$ & Difference [95\% Cl] & $P$ value $^{A}$ \\
\hline \multirow[t]{2}{*}{ Sex } & Male & 319 & $4.87[4.69,5.06]$ & Base & 0.8332 \\
\hline & Female & 313 & $4.90[4.73,5.07]$ & $0.03[-0.23,0.28]$ & \\
\hline \multirow[t]{3}{*}{ Age (years) } & $<5$ & 28 & $4.55[3.94,5.16]$ & Base & 0.0104 \\
\hline & $5-14$ & 99 & $4.72[4.42,5.02]$ & $0.17[-0.72,1.02]$ & \\
\hline & $15-40$ & 381 & $4.79[4.63,4.96]$ & $0.24[-0.57,1.06]$ & \\
\hline & $\geq 5$ days & 460 & $4.63[4.48,4.78]$ & $-0.95[-1.22,-0.67]$ & \\
\hline \multirow[t]{2}{*}{ Outcome } & Survivors & 379 & $4.02[3.89,4.15]$ & Base & $2.70 \times 10^{-77}$ \\
\hline & Nonsurvivors & 253 & $6.18[6.04,6.32]$ & $2.16[1.96,2.36]$ & \\
\hline Total & & 632 & $4.89[4.76,5.02]$ & - & - \\
\hline
\end{tabular}

ADetermined using a 2-tailed unpaired equal variance $t$ test for sex and survival. For age and time since onset of symptoms (sampling), the significance was determined using a linear regression of $\log _{10} \mathrm{CEQ} / \mathrm{ml}$ vs. age in years or $\log _{10} \mathrm{CEQ} / \mathrm{ml}$ vs. time since onset of symptoms.

same 632 patients by viral load and calculated the CFRs for each group (Supplemental Figure 2). Viremia was arbitrarily classified as low, intermediate, or high $(<4.24,4.24-6.94$, and $>6.94 \mathrm{GEQ} / \mathrm{ml}$, respectively, corresponding to Ct values of $>30,20-30$, and $<20$ ). Only samples from July (Figure 2A) and November (Figure 2B) were investigated in order to evaluate differences observed in patients between the beginning and the end of data collection.

The analysis revealed that the CFR for July was significantly higher $(52.7 \%$, Figure $2 \mathrm{~A})$, than for November $(30.9 \%$, Figure 2B) $\left(\chi^{2}[1]=22.862, P=1.7 \times 10^{-6}\right)$. However, the differences in CFR for each viral load cluster were not significantly different between July and November 2014 (high viral load: $\chi^{2}[2]=0.715$, $P=0.699$; medium viral load: $\chi^{2}[2]=4.518, P=0.104$; low viral load $\left.\chi^{2}[2]=2.650, P=0.266\right)$. This is known as Simpson's paradox: the difference in the overall rates is a result of the fact that the low and intermediate viral loads are overrepresented in November (Supplemental Figure 3$)$. However, the CFRs by viral load clusters were significantly different from each other within each month (July: $\chi^{2}[2]=49.432$, $P=1.8 \times 10^{-11}$; November: $\chi^{2}[2]=115.67, P \leq 2.2 \times 10^{-16}$ ), indicating that patients with different levels of viremia died at differing rates. The Marascuillo procedure for comparing multiple proportions showed that all 3 levels of viremia had statistically different fatality rates for both July and November. The analysis revealed that viral loads above $6.94 \log _{10} \mathrm{GEQ} / \mathrm{ml}$ of blood on admission led to fatal outcomes in $89.1 \%$ of cases (95\% CI, 81.3-96.9). In contrast, viral loads lower than $4.24 \log _{10}$ GEQ/ml of blood on admission were associated with death in $7.4 \%$ of cases (95\% CI, 5.8-9) (Supplemental Figure 2). Similarly, there was a difference in outcomes between patients with high and intermediate viremia, and between intermediate and low viremia, with the higher viremia linked to a higher fatality rate.

To identify the optimal threshold of viremia, which would be predictive of nonsurvival for each month (Figure 2C), a receiver operating characteristic analysis (ROC analysis) was performed. Three important summary values of ROC analysis are the sensitivity, the specificity, and the positive predictive value (PPV). The sensitivity represents the fraction of true positives out of the condition positives (true positives plus false negatives), the specificity refers to the fraction of true negatives out of the condition negatives (true negatives plus false positives), and the PPV represents the fraction of true positives out of the test positives (true positives plus false positives). The optimal viral load threshold that was predictive of nonsurvival for the month of July was $5.860 \log _{10}$ GEQ/ml of blood (sensitivity: 0.694 [95\% CI, 0.554-0.805]; specificity: 0.864 [95\% CI, 0.751-0.932]; PPV: 0.810 [95\% CI, 0.690-0.930]) (Supplemental Table 3). For November, the threshold value was $4.672 \log _{10} \mathrm{GEQ} / \mathrm{ml}$ of blood (sensitivity: 0.752 [95\% CI, 0.670-0.818]; specificity: 0.910 [95\% CI, 0.814-0.961]; PPV: 0.942 [95\% CI, 0.897-0.987]) (Supplemental Table 3). This later threshold could appropriately identify $75.2 \%$ of individuals who died and $91 \%$ of the individuals who survived. Out of the individuals predicted not to survive, $94.2 \%$ died.

Effect of age on mortality. Mortality rates were compared between age groups using the $\chi^{2}$ test $\left(\chi^{2}[3]=18.888, P=0.00029\right)$, followed by the Marascuillo procedure for comparing multiple proportions. It was observed that there was a marked difference in mortality between the 5- to 14-year-olds and the $>40$-year-olds, as well as between the 15 - to 40 -year-olds and the $>40$-year-olds.

Individual patient viral kinetics and survival. Patients who presented to the EMC and initially tested positive for EBOV were kept under observation and sampled to monitor disease progression and convalescence according to uneven clinical management requirements. To assess if the viral load kinetics of individual patients correlated with survival, we plotted the viremia of survivors and nonsurvivors over the course of disease for each month (Figure 3, $\mathrm{A}-\mathrm{E})$. It should be noted that nonsurvivors often have very few time points due to sudden mortality, thereby limiting this analysis (10). Therefore, survivors who had $\geq 5$ samples and nonsurvivors with $\geq 2$ samples were used in our analysis, for a total of 40 patients. The various viral profiles did not reveal meaningful trends that would allow for outcome prediction on an individual-patient basis. This result is consistent with data previously reported regarding the Sudan virus (SUDV) outbreak in Gulu, Uganda (10). During that outbreak, it was determined that, while viremia in survivors was often found to start high and decrease over time, viremia in nonsurvivors varied considerably. Interestingly, the second measure- 

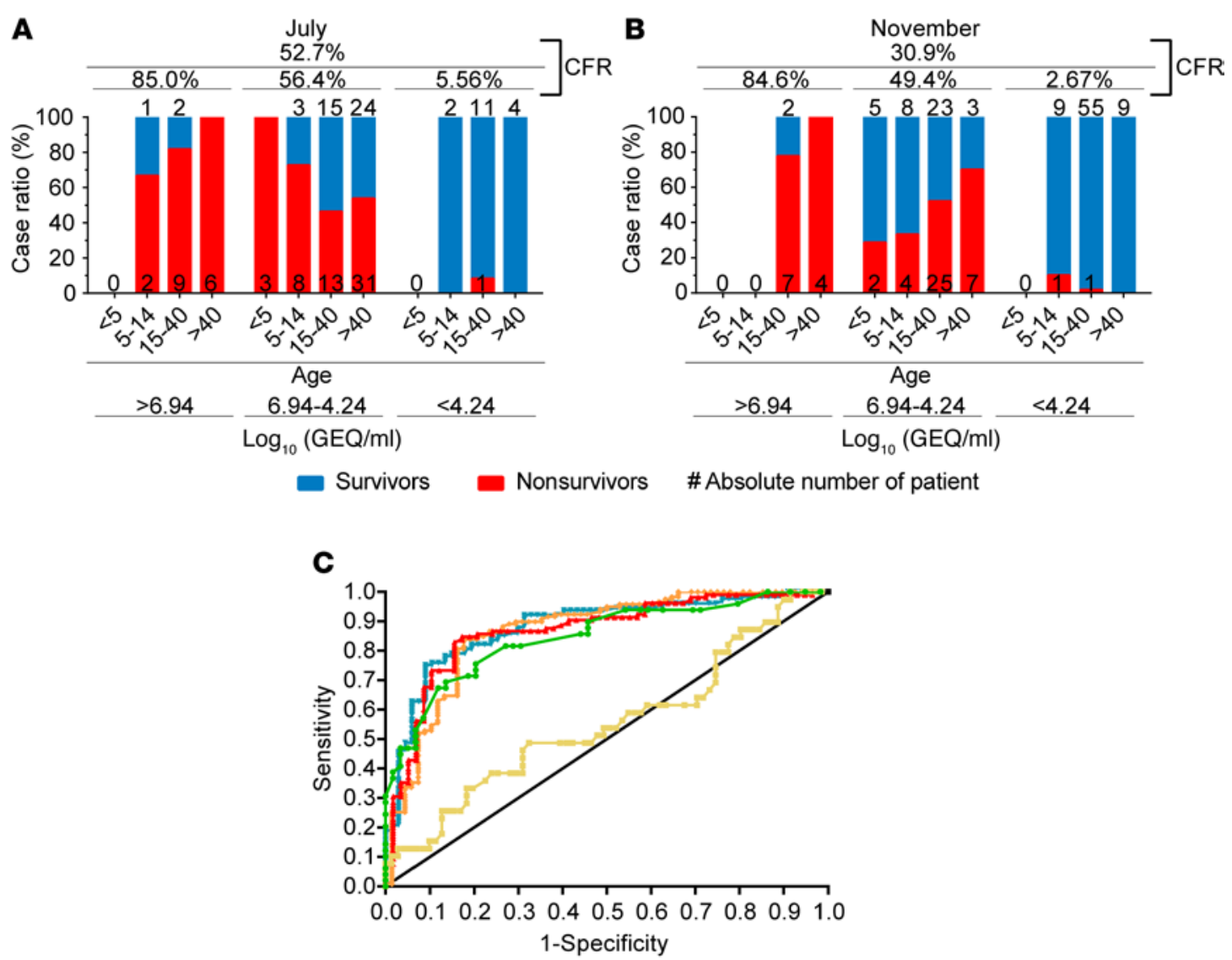

Figure 2. Correlation between viral load and outcome for the months of July and November. The CFR (\%) and the case survival rate (CSR, \%) are stacked, divided by viral clusters of low (<4.24), intermediate (4.24-6.94) and high (>6.24) viremia and subdivided by age groups (below 5, between 5 and 14 , between 15 and 40, and above 40 years old). (A and B) Months of July $(n=93)(\mathbf{A})$ and November $(n=165)$ (B) are represented. Survivors are shown in blue and nonsurvivors are shown in red. (C) Sensitivity and 1 minus specificity (ROC curves) are shown at various threshold values of viral loads for each month of the outbreak in Kailahun. (July: green, $n=47$; August: yellow, $n=81$; September: red, $n=134$; October: orange, $n=159$; November: blue, $n=164$ )

ment of viremia of patients who experienced a fatal outcome was lower than the first measurement in 4 of the 10 cases versus for 21 of 26 survivors - a significant difference $\left(\chi^{2}[1]=8.47, P=0.0036\right)$

Change in initial viral load over time. To further examine the relationship between the viral load and outbreak progression, as well as viral load and mortality, a linear model was developed (Figure 4A; see Annex I in the supplemental material). This model allowed to account for any effect that the time since onset of symptoms may have had on viremia. Two additional potential predictors (sex and age) were also included in the model and were found to be unrelated to viremia in this dataset, once other predictors were accounted for (see Annex I in the supplemental material). The effect of time since the beginning of the outbreak was found to be more accurately modeled as a quadratic relationship, suggesting that the viral loads were actually increasing toward the end of the data-collection period (Figure 4B). However, there is still a negative linear component to the regression, which explains why the simple linear regression in Figure $1 \mathrm{~A}$ had a nonzero negative slope. The difference in viral load for survivors was $1.6 \log _{10} \mathrm{GEQ} / \mathrm{ml}$ of blood lower than nonsurvivors. This difference increases by $0.073 \log _{10} \mathrm{GEQ} / \mathrm{ml}$ of blood with every day after the onset of symptoms (Figure 4C).

Survival correlates with population immunity. Initial viral loads of patients were higher in July when compared with November in
Kailahun, Sierra Leone (Figure $1 \mathrm{~A}$ and Supplemental Figure 1). Interestingly, the CFRs for each month followed an overall downward trend. The monthly CFRs were $52.7 \%, 67.4 \%, 31.8 \%, 32.9 \%$, and $30.9 \%$, respectively (Figure 5). A $\chi^{2}$ test for independence revealed an association between month and CFR $\left(\chi^{2}=118.37\right.$, degrees of freedom $=9, P<2.2 \times 10^{-16}$ ). The significant $\chi^{2}$ was followed by the Marascuillo procedure to compare multiple proportions, which showed significant differences in CFR between July and the months of September, October, and November, as well as between August and the months of September, October, and November (Supplemental Figure 4). It was hypothesized that increasing immunity in the population could be one of the contributors to the lower viral loads by reaching a herd immunity threshold as the outbreak progressed. To evaluate this hypothesis, the seroprevalence of EBOV-specific IgG was analyzed. Ninety-four patients who presented at the EMC for diagnostic testing, but who were determined to be negative for EBOV by reverse transcription PCR (RT-PCR) between July and November 2014, were assessed for EBOV-specific IgG. Out of these 94 patients, 46 reported history of contact with a sick person or funeral exposure; 17 reported no exposure to an infected individual or attendance to a funeral; and, for the 31 other individuals, the information was not available. Forty-five of these 94 patients (47.9\%) had detectable IgG antibodies against EBOV (Supplemental Table 5). 
A

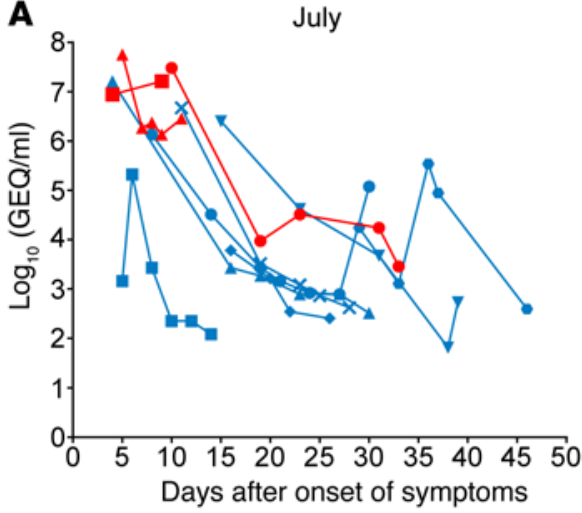

B

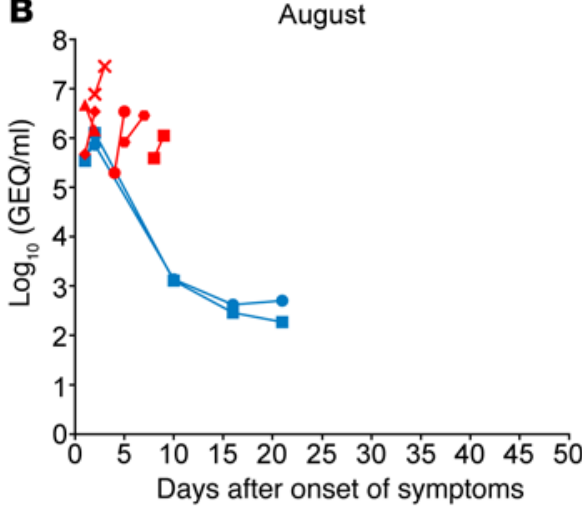

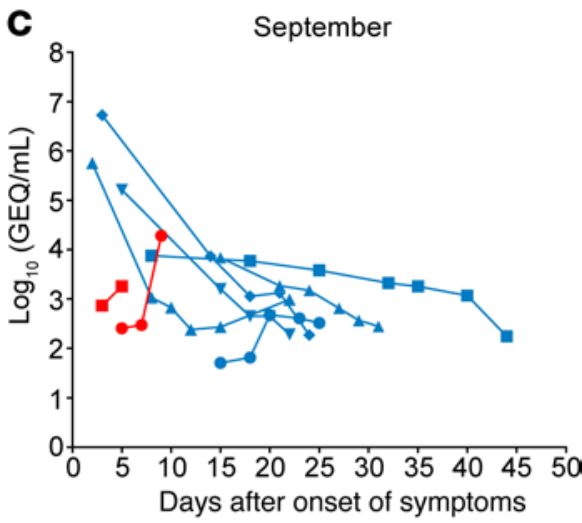

D

October

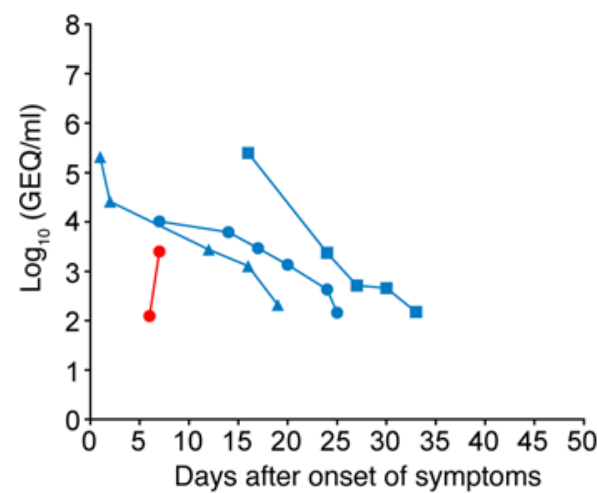

E

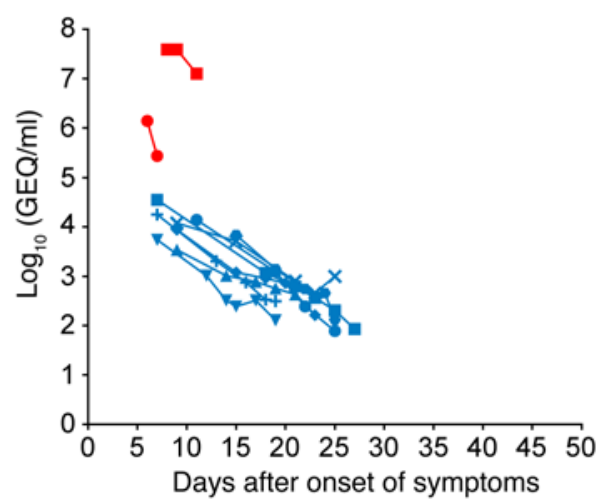

Figure 3. Viral kinetics of individual patients throughout the outbreak. The viremia of individual patients was graphically represented by number of days after onset of symptoms. (A-E)Patients were divided by months of the outbreak in Kailahun, Sierra Leone, in July $(n=10)(\mathbf{A})$, August $(n=8)(\mathbf{B})$, September $(n=8)(\mathbf{C})$, October $(n=4)(\mathbf{D})$, and November $(n=10)(\mathbf{E})$. Each symbol for a given panel represents a single patient. Survivors are shown in blue, and nonsurvivors are shown in red.

The analysis indicated that immunity in EBOV-negative patients was increasing, concurrent with decreasing viral loads in infected patients (Figure 5). In July, the EBOV seroprevalence rate was $14.3 \%$ (1 patient out of 7;95\% CI, 0\%-40.2\%), while in September, the rate increased to $55.9 \%$ (19 patients out of 34 ; 95\% CI, $44.3 \%-67.4 \%$ ). This trend continued through October (12 patients out of $25 ; 48.0 \%$; 95\% CI, 28.4\%-67.6\%) and November, where 13 patients out of 28 (46.4\%; 95\% CI, 27.9\%-64.9\%) were seropositive for EBOVIgG. Linear regression analysis established that the increase in the monthly immunity was significant $\left(\mathrm{F}_{1,2}=20.29, P=0.0459\right)$. Unfortunately, samples from late August 2014 were unavailable for testing, which prevented us from further detailing the crossover in CFR and EBOV seroprevalence.

\section{Discussion}

The current findings suggest that the viral load of a patient on arrival to the EMC is statistically predictive of outcome. Individual viral kinetics were only associated with outcome in regards to the second viral load determination in relation to the first one (e.g., nonsurvivors tend to show higher second measurements). Interestingly, $1 / 3$ of the patients who experienced a fatal outcome were not following this trend - a counterintuitive observation, since virus clearance should be associated with survival. Presence of one or many co-morbidities or irreparable tissue damage resulting from virus replication could explain this phenomenon. This is in line with a previous study (10), which showed, in the context of SUDV infections, that predicting an outcome based on an overall viralload profile was not accurate. However, this could also be explained by an insufficient data set, where many nonsurvivors had few data points available. Therefore, this analysis cannot rule out that individual kinetics could be associated with clinical outcome.

Linear modeling of the initial viremia as a function of (i) the time between onset of symptoms and sampling, (ii) the time since the beginning of data collection, and (iii) the outcome of the patients allowed for the analysis of subtler trends in the viremia. From the start of the outbreak, there was a difference in viremia of about 1.6 log-units (95\% CI, 1.3-1.9) between survivors and nonsurvivors, and this difference increased as the outbreak progressed. However, a quadratic component to the relation between viremia and the time since the beginning of data collection also emerged, which suggests that the viremia was not decreasing during the months of October and November. This explains why the decrease in viremia between July 1 and October 31 (or November 30) was lower than the decrease between July 1 and September 30. As expected, the difference in viremia was also increasing with the time since onset of symptoms. These results suggest that, for fatal cases, the time since onset of symptoms had a neutral to positive effect on viremia, whereas, for the survivors, the more time 
A
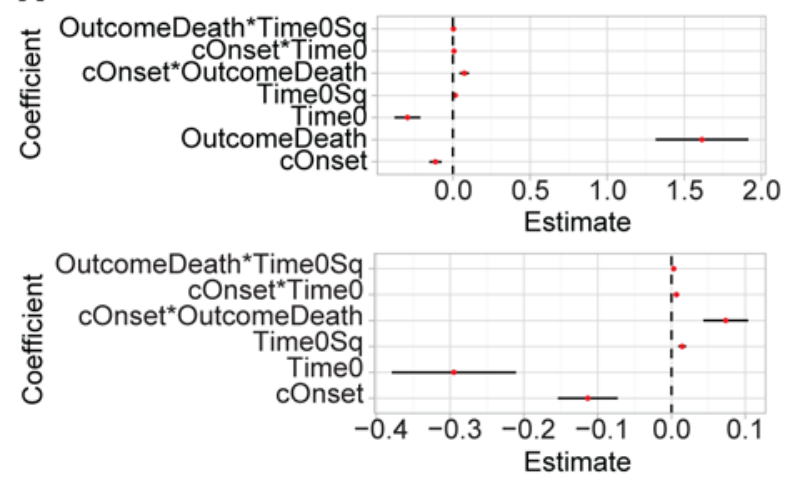

C

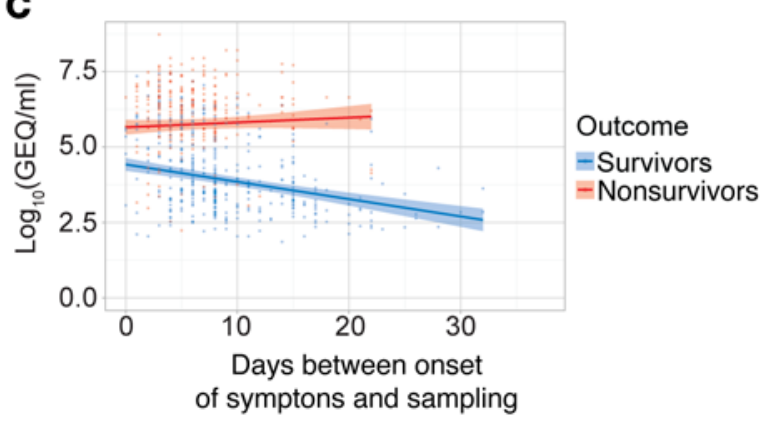

there was between onset of symptoms and sampling, the lower the viremia. Overall, this model supports other analyses in concluding that there was indeed a decrease in initial viremia, even when we account for variation in time since onset of symptoms.

An intriguing finding is the correlation between the observed increase in EBOV-specific antibodies in the population and the decrease in initial viral loads of infected patients. Previous work conducted by the Lassa Diagnostic Laboratory at the Kenema General Hospital (KGH), Sierra Leone, between October 2006 and October 2008 revealed that 19 of the 220 (8.6\%) serum samples tested during that period had an antibody response against EBOV (11). This number seems to be in accordance with another study conducted in rural areas of Gabon, a country that had been affected by 5 outbreaks of EBOV at the time of this serological survey. The Gabonese study, conducted from 2005-2008, established that 667 participants out of 4,349 (15.3\%) were seropositive for EBOV-specific IgG (12). The higher seroprevalence observed in this last study compared with the one in Sierra Leone, despite similar sampling times, could potentially be explained by the higher occurrence of outbreaks in Gabon, leading to a larger fraction of the population being exposed to EBOV. A second study conducted at the KGH, from June 2011 to March 2014, analyzed a subset of 242 serum samples (13). Of those samples, between $16(6.8 \%)$ and $53(22 \%)$ were seropositive for IgG against EBOV, depending on the stringency of the cut-off applied to the assay. The findings presented here with an immunity of $14 \%$ for the month of July are in accordance with this later study, despite the few data points available in the present analysis in July. Overall, these serological surveys demonstrate that EBOV-specific IgG can be found in the population outside of an epidemic context, but also in regions where an EBOV outbreak has never been documented. It should be noted that genomic analysis had postulated

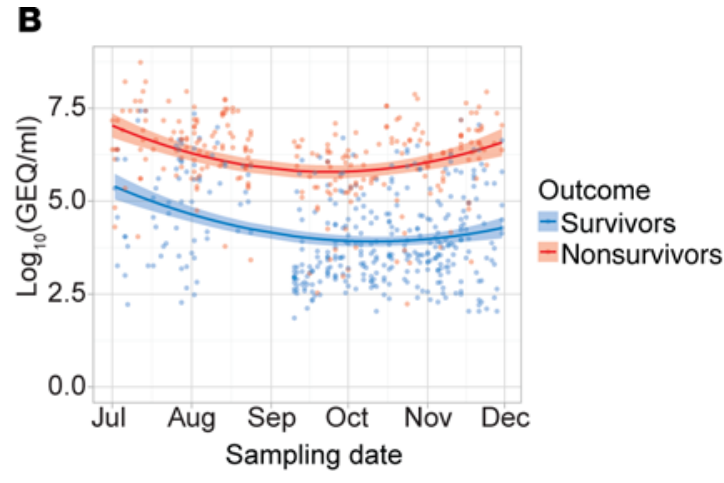

Figure 4. Linear model of the viremia. Estimating the difference between survivors and nonsurvivors, as well as the effect of outbreak duration on initial viremia. $(n=632)$. (A) Regression coefficients for the Makona outbreak; point estimates and 95\% Cl. Upper panel: All coefficients. Lower panel: Excludes from the graphical representation the outcome to show the smaller coefficients. (B) Effect of outbreak duration for survivors and nonsurvivors. (Showing best line and $95 \% \mathrm{CI}$ ). (C) Effect of time since onset of symptoms at sampling time for survivors and nonsurvivors (showing best line and $95 \% \mathrm{Cl}$ ).

that the currently circulating strain may have diverged and been introduced to West Africa in 2004 (7). Thus, it is tempting to speculate that occasional exposure to this virus, which would generate a low level of population immunity, may have occurred prior to the current outbreak. A study published in 2000 suggested that asymptomatic, replicative Ebola infection can occur in humans and that a fraction of those individuals can develop an antibody response (14). Similar to the serosurveys mentioned above, the one conducted in the current study could only use a small number of samples relative to the number of patients used for viremia assessment. In addition, as in previous studies, it is important to mention that the results were generated from patients reporting at a clinic with symptoms (in the present case at the EMC) and not from a random sample of the community. In this case, specifically, the patients were sick but EBOV-negative by quantitative PCR (qPCR), which may have introduced a bias and not necessarily represent the immune status of the general population. Therefore, although interesting, interpretation from these observations should be taken with caution until data from an extended number of samples are available.

Overall, the present data suggest a significant decrease in the viral loads over time in patients in their initial screen for EBOV upon arrival to the EMC in Kailahun, Sierra Leone. Hypotheses to explain this decrease are numerous and not mutually exclusive. As the outbreak progressed and more cases arose, the awareness of the disease among the population could have increased, and in the event that one family member fell ill, every household member could seek early medical care or actively reduce their exposure by limiting their contacts with sick individuals. However, the fact that there is no statistically significant difference in time between onset of symptoms and initial diagnostic sampling suggests that faster reporting to the EMC is not supported by the current data. 


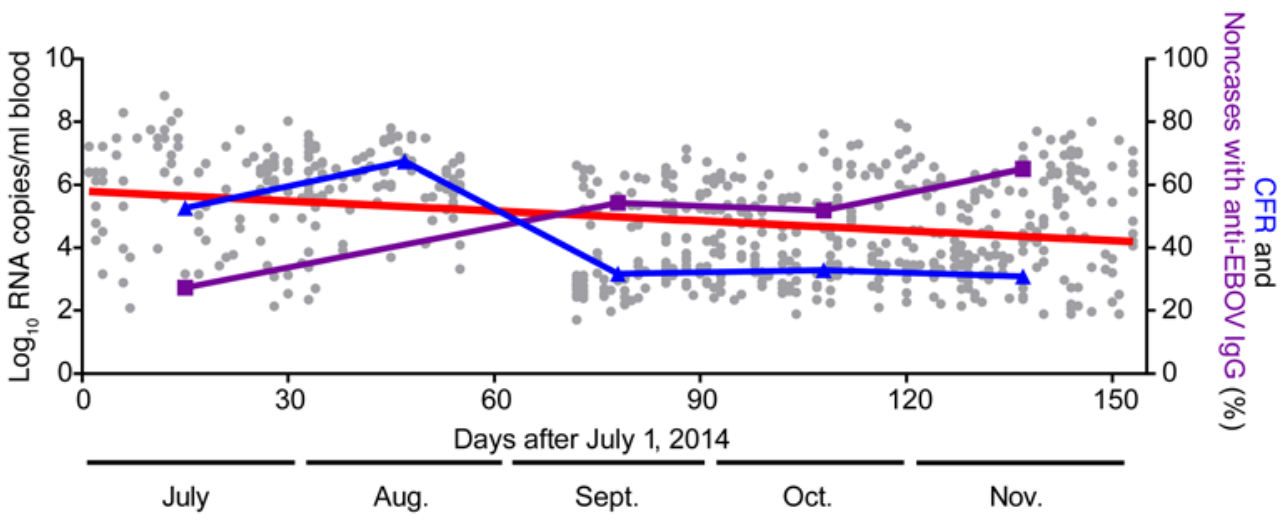

Figure 5. Evolution of the CFRs and immunity in the population. Monthly CFRs (blue triangles) and percentage of the tested population with an immunity to EBOV (purple squares) are overlaid with the initial viremia of the selected 632 patients (gray circles). Linear regression of the viremia is shown in red $(n=632)$.
It also remains possible that the decrease is due to a detection bias. As the outbreak progressed, a growing spectrum of cases were identified and tested, whereas early in the outbreak, it is possible that more of the severe cases (with expected higher viremia) were detected and tested. Anecdotally, however, many individuals are still refusing to acknowledge the disease and prefer not to report to an EMC. Even now, over a year after the outbreak was officially declared, many security incidents are still being reported, indicating that there is still a lack of trust in certain populations toward healthcare facilities and outbreak responders. Another possibility is the existence of patients with atypical infections, who do not show traditional symptoms of EVD, such as subclinical infections. The individuals would be far more mobile than patients with a more typical infection. As such, individuals with an atypical infection could spread the virus ahead of the wave of severe infections and increase the levels of immunity in the population. Once the level of immunity reaches a critical threshold, fatality rates and viremia would go down, as observed in the present study.

It is fair to assume that, as the outbreak progresses through time, it is also progressing in geographical distribution. As the outbreak moved away from the epicenter, where the EMC was located, it is possible that severely afflicted patients were unable to complete or even undertake the journey to the EMC, thereby filtering out the patients with the highest viremia. However, given that highly viremic patients tend to be very contagious, a large flareup would have been expected, which did not occur. Future work will need to assess the impact of this parameter, among others, on outbreak progression. Finally, another hypothesis to consider is genetic variation of the virus. As described recently (15), isolates collected in Kailahun from July to November 2014 were shown to have become more phylogenetically and genetically diverse, resulting in the emergence of a multitude of novel lineages. Even though this diversification has not been linked to any phenotype of the virus (16), it could be the result of an adaptation of EBOV to its host, resulting in slower replication and a lower viremia.

Overall, the current study offers a detailed view on viral loads in relation to epidemiological data from a highly active site of EBOV transmission and spread. These data could inform the development of vaccine, therapeutics, and importantly, the design of optimal clinical trials by associating promising clinical modalities in a way to better benefit a subgroup of individuals with the highest predicted CFR.

\section{Methods}

Study population. From July to November 2014, the mobile laboratory operated by the PHAC was deployed to provide diagnostic testing support at an EMC in the Kailahun district of Sierra Leone, managed by MSF. During these 5 months, over 2,700 samples from about 1,200 patients were analyzed. December was excluded from the analysis, as there were only 2 positive cases during this month. Patients were screened on arrival at the EMC and relocated to the relevant areas of the EMC. Patients who tested positive for EBOV were kept at the EMC to receive care until final outcome (death or convalescence), while the ones who were found negative were released. The mobile laboratory staff was evacuated for safety reasons on August 25, 2014; the laboratory was reopened on September 10, 2014.

Procedures. Samples were received by the onsite laboratory and inactivated using a previously published method (17) inside a flexible film, negative pressure isolator using Rapid Containment Kit (Germfree Laboratories) followed by RNA extraction and purification. qPCR assays targeting the Ebola polymerase (L) and nucleoprotein (NP) genes were used to detect the presence of EBOV using Lightcycler 480 RNA Master Hydrolysis reagents (Roche Diagnostics) according to manufacturer's instructions. Primers and probes were as follows: ZEBOV LF (5'-CAGCCAGCAATTTCTTCCAT), ZEBOV LR (5'-TTTCGGTTGCTGTTTCTGTG), ZEBOV LP1 (FAM-ATCATTGGC/ZEN/ RTACTGGAGGAGCAG-BHQ1), ZEBOV LP2 (FAMTCATTGGCG/ ZEN/TACTGGAGGAGCAGG-BHQ1), ZEBOV NPF (5'-TGCCGACGACGAGACGT), ZEBOV NP2 (5'-CGTCCCTGTCCTGTTCTTCAT), and ZEBOV NPP (FAM-AGYCTTCCG/ZEN/CCCTTGGAGTCAGA). Probes were obtained from IDT Labs and contained an internal quencher (/ZEN/), as indicated.

Assays were performed on the Lightcycler Nano platform (Roche Diagnostics). Primary testing focused on EBOV diagnosis using cutoff cycle threshold (CT) values for positive, equivocal, and negative of $\leq 37,37.1-40$, and $>40$, respectively. The assays detected EBOV at approximately 10 genome equivalents/reaction. Differential diagnostics were also offered for Lassa virus (18) and Plasmodium species (19). As an internal control for extraction and amplification procedures, MS2 phage was added to each sample as previously described (20) to ensure that the extraction, PCR setup, and run parameters did not change between operators or over time. The data shown was generated from EDTA blood samples collected at the MSF EMC. CT values were converted to GEQ/ml based on the average of 10 replicates of an in-house laboratory standard curve (Supplemental Table 1). 
IgG ELISA were performed using the Crocodile ELISA miniWorkstation (5-inone) (Titertek-Berthold). Polystyrene microtitre plates (Costar, Corning Inc.) were coated with $50 \mathrm{ng} /$ well/100 $\mu \mathrm{l}$ of sucrose purified inactivated EBOV in PBS overnight at $4^{\circ} \mathrm{C}$. Sucrose purified inactivated Marburg virus (MARV) was used as a negative control antigen (Ag). Plates were washed with PBS containing 0.1\% Tween-20 (washing buffer), then blocked with $200 \mu \mathrm{l}$ PBS-5\% skim milk for $15 \mathrm{~min}$ utes at $37^{\circ} \mathrm{C}$, before sera diluted in blocking solution $(33 \mu \mathrm{l})$ was added for 1 hour at $37^{\circ} \mathrm{C}$. After washing, $100 \mu$ l of detection antibody (Peroxidaseconjugated goat anti-human IgG) (diluted 1:2,000 in blocking solution) was added for 1 hour at $37^{\circ} \mathrm{C}$. Subsequently, plates were washed, and $100 \mu \mathrm{l}$ of substrate (2,2'-azino-bis[3-ethylbenzothiazoline-6-sulphonic acid] [ABTS]; KPL) was added to each well for 20 minutes at $37^{\circ} \mathrm{C}$, before reading on a microplate reader at $405 \mathrm{~nm}$. A sample was positive when the absorbance minus background was higher than the mean plus 4 standard derivations of the serum pool of 20 normal people.

Statistics. The overall linear regression of viremia over time, $t$ tests, and ANOVA were performed using GraphPad Prism version 6.05. For $t$ tests, Gaussian distribution was assumed and 2-tailed, unpaired equal variance $t$ tests were conducted at a confidence level of $95 \%$. Ordinary 1-way ANOVAs with Tukey's multiple comparisons test (single pooled variance) were conducted at a confidence level of $95 \%$.

In order to assess the difference in viremia over time and between survivors and nonsurvivors, we used a linear model to account for other variables that may have impacted the viral loads. The predictors we explored were: (i) the time (in days) since July 1, 2014; (ii) the time (in days) between the initial sample and onset of symptoms (the onset of symptoms was estimated by the patients and physicians); (iii) the age of the patient; and (iv) the sex of the patient. The significance threshold for including the individual main effects in the model was 0.25 , and the significance threshold for keeping a predictor in the full model was 0.1 . The linearity of the relationships was verified graphically; the time since July 1, 2014, was the only nonlinear predictor. The differences in CFRs between multiple groups were tested by the $\chi^{2}$ test followed by the Marascuillo procedure, if the $\chi^{2}$ test was significant. The data cleaning, preparation, and analysis were performed using Revolution R open (based on R version 3.1.2, with a CRAN snapshot from April 30, 2015; Revolution Analytics). A P value less than 0.05 was considered significant.

Study approval. This work was conducted as part of the international public health response to help with the containment of the outbreak in Sierra Leone, and therefore, informed consent was not obtained from individual patients. Ethics approval was obtained from the Government of Sierra Leone Ethic Review Board in Freetown to use, analyze, and publish controlled, unidentified, anonymous data collected during diagnostic testing. This study met the standards set by the independent MSF Ethics Review Board for retrospective analyses of routinely collected programmatic data (21).

\section{Author contributions}

MADLV, GC, JA, AS, and GPK designed the research study. GC, XQ, JIB, JG, KL, DKK, BK, AG, DK, JES, and GPK acquired the data. MADLV, JA, and SK analyzed the data. MADLV, JA, and GPK wrote the manuscript. MADLV, GC, JA, RAK, AW, AS, ADC, GI, MVH, and GPK edited the manuscript.

\section{Acknowledgments}

The authors thank the government of Sierra Leone for their collaboration, MSF for their deployment and field assistance, and everyone who was deployed and helped, even remotely, in generating the data used in this article. This work was supported by the PHAC. M.A. de La Vega is the recipient of a Master Research Award from the Canadian Institute for Health Research (CIHR).

Address correspondence to: Gary P. Kobinger, Special Pathogens Program, National Microbiology Laboratory, PHAC, 1015 Arlington Street, Winnipeg, MB, R3E 3R2 Canada. Phone: 204.784.5923; E-mail: gary.kobinger@phac-aspc.gc.ca.
1. Kuhn JH, et a. Filovirus RefSeq entries: evaluation and selection of filovirus type variants, type sequences, and names. Viruses. 2014;6(9):3663-3682.

2. Kuhn JH, Jahrling PB. Clarification and guidance on the proper usage of virus and virus species names. Arch Virol. 2010;155(4):445-453.

3. Feldmann H, Geisbert TW. Ebola haemorrhagic fever. Lancet. 2011;377(9768):849-862.

4. Ansari AA. Clinical features and pathobiology of Ebolavirus infection. J Autoimmun. 2014;55:1-9.

5. Baize S, et al. Emergence of Zaire Ebola virus disease in Guinea. $N$ Engl J Med. 2014;371(15):1418-1425.

6. Dudas G, Rambaut A. Phylogenetic analysis of Guinea 2014 EBOV Ebolavirus outbreak. PLoS Curr. 2014;6.

7. Gire SK, et al. Genomic surveillance elucidates Ebola virus origin and transmission during the 2014 outbreak. Science. 2014;345(6202):1369-1372.

8. Ebola virus disease in Liberia [press release]. Disease Outbreak News. World Health Organization; March 30, 2014.

9. Ebola virus disease, West Africa - update [press release]. Disease Outbreak News. Guinea, Africa; World Health Organization; May 24, 2014.

10. Towner JS, et al. Rapid diagnosis of Ebola hemorrhagic fever by reverse transcription-PCR in an outbreak setting and assessment of patient viral load as a predictor of outcome. J Virol. 2004;78(8):4330-4341.

11. Schoepp RJ, Rossi CA, Khan SH, Goba A, Fair JN. Undiagnosed acute viral febrile illnesses, Sierra Leone. Emerg Infect Dis. 2014;20(7):1176-1182.

12. Becquart $P$, et al. High prevalence of both humoral and cellular immunity to Zaire ebolavirus among rural populations in Gabon. PLoS One. 2010;5(2):e9126.

13. Boisen ML, et al., Multiple circulating infections can mimic the early stages of viral hemorrhagic fevers and possible human exposure to filoviruses in Sierra Leone prior to the 2014 outbreak. Viral Immunol. 2015;28(1):19-31.

14. Leroy EM, et al. Human asymptomatic Ebola infection and strong inflammatory response. Lancet. 2000;355(9222):2210-2215.

15. Tong YG, et al. Genetic diversity and evolutionary dynamics of Ebola virus in Sierra Leone. Nature. 2015;524(7563):93-96.
16. Olabode AS, Jiang X, Robertson DL, Lovell SC. Ebolavirus is evolving but not changing: no evidence for functional change in EBOV from 1976 to the 2014 outbreak. Virology. 2015;482:202-207.

17. Grolla A, Mehedi M, Lindsay R, Bosio C, Duse A, Feldmann H. Enhanced detection of Rift Valley fever virus using molecular assays on whole blood samples. J Clin Virol. 2012;54(4):313-317.

18. Demby AH, Chamberlain J, Brown DW, Clegg CS. Early diagnosis of Lassa fever by reverse transcription-PCR. JClin Microbiol. 1994;32(12):2898-2903.

19. Lee MA, et al. Real-time fluorescence-based PCR for detection of malaria parasites. JClin Microbiol. 2002;40(11):4343-4345.

20. Dreier J, Störmer M, Kleesiek K. Use of bacteriophage MS2 as an internal control in viral reverse transcription-PCR assays. J Clin Microbiol. 2005;43(9):4551-4557.

21. Medecins Sans Frontieres. MSF Ethics Review Board Standard Operating Procedures. MSF Web site. http://fieldresearch.msf.org/msf/ handle/10144/294968. Updated February 12, 2013. Accessed October 5, 2015. 